\title{
Local Turbulence and the Dipole Anisotropy of Galactic Cosmic Rays
}

\author{
Yoann Genolini $^{a, *}$ and Markus Ahlers ${ }^{a}$ \\ ${ }^{a}$ Niels Bohr International Academy, Niels Bohr Institute, University of Copenhagen, \\ Blegdamsvej 17, 2100 Copenhagen, Denmark \\ E-mail: yoann.genolini@nbi.ku.dk, markus.ahlers@nbi.ku.dk
}

The dipole anisotropy of multi-TeV cosmic rays exhibits a strong energy dependence that is at odds with the predictions of standard isotropic diffusion models. It has been argued that the observed variation in amplitude and phase is a consequence of the global distribution of cosmic ray sources in combination with anisotropic diffusion in our local environment. For a quantitative description of this effect it is necessary to understand the complicated interplay of cosmic ray diffusion on local and global scales. In this work we study the impact of isotropic magnetic turbulence realisations on cosmic-ray propagation and anisotropy. We define a novel methodology that allows us to quantify generic properties of local and global diffusion with the help of test-particle simulations. We confirm the emergence of local anisotropic diffusion that leads to an alignment of the cosmic ray dipole with the local magnetic field and a reduction of its amplitude in perpendicular directions. We discuss the phenomenological consequences of these findings.

$3^{\text {th }}$ International Cosmic Ray Conference (ICRC 2021)

July 12 th - 23rd, 2021

Online - Berlin, Germany

\footnotetext{
${ }^{*}$ Presenter
} 


\section{Introduction}

Upon their arrival at Earth, cosmic rays (CRs) will have experienced repeated deflections in magnetic fields since their departure in distant Galactic or extragalactic sources. The spatial variation of these magnetic fields in terms of strength and orientation leads to a random walk of charged particles. The transport of CRs in the presence of turbulent magnetic fields can be effectively described as a diffusive process [1]. The effect of random scattering in turbulent magnetic fields is encapsulated in the diffusion tensor $\mathbf{K}$. In standard diffusion theory, the first order correction to the isotropic distribution of cosmic ray arrival direction is a small dipole anisotropy that scales with the local CR gradient, $\nabla n$, according to Fick's law. The phase and strength of this dipole is expected to be a combined effect of the relative motion of the solar system with respect to the frame where CRs are isotropic [2], the distribution of cosmic ray sources [3-5], the characteristics of turbulent magnetic fields as well as the strength and orientation of local regular magnetic fields [6-8].

In recent years many experiments achieved the necessary level of statistics to be able to find anisotropies in cosmic ray arrival directions up to a level of 1 part in 1,000; see Refs. [9-12]. The left panel of Fig. 1 shows the dipole anisotropy data from various experiments in the TeV to multi-PeV energy range. The data is shown in terms of the amplitude and right ascension (RA) phase after the projection of the dipole vector onto the equatorial plane. One can notice that the (projected) dipole exhibits a strong energy dependence in the multi-TeV to $\mathrm{PeV}$ energy range that seems to be at odds with the predictions of standard isotropic diffusion models indicated by the dashed lines in the plot. However, it has been argued that the observed variations in amplitude and phase are a natural consequence of the global distribution of cosmic ray sources in combination with anisotropic diffusion in our local environment [6-8]. These arguments are consistent with the observed magnetic field in our local Galactic environment. However, for a quantitative description of this effect it is necessary to understand the complicated interplay of cosmic ray diffusion on local and global scales.

In these proceedings we study the effect of the imprint of the local realisation of magnetic turbulence on the magnitude and orientation of cosmic ray dipole anisotropy. Our discussion will be based on the formalism of [13] that allows to study the power of anisotropies in cosmic ray arrival directions induced by a large scale cosmic ray gradient. While the size and orientation of the local cosmic ray gradient is determined by source locations and spatially averaged diffusion properties, the local anisotropy has to account for the gradual transition of cosmic ray propagation from the local ballistic, over the intermediate laminar to the asymptotic diffusive regime.

\section{Local Dipole Anisotropy}

After their release in distant sources, cosmic rays experience deflections in magnetic fields before arrival at Earth. In the following it is understood that the magnetic field, $\mathbf{B}=\mathbf{B}_{0}+\delta \mathbf{B}$, has a strong turbulent component $\delta \mathbf{B}$ on top of a constant background field $\mathbf{B}_{0}$. In the case of static magnetic fields, the evolution of the CR phase-space density (PSD), $f(t, \mathbf{r}, \mathbf{p})$, follows Liouville's theorem, $\dot{f}(t, \mathbf{r}, \mathbf{p})=0$.

After sufficiently long time-scales, the repeated scattering of cosmic rays in magnetic turbulence will have effectively wiped out most of its memory of its prior path. The only relevant quantities 

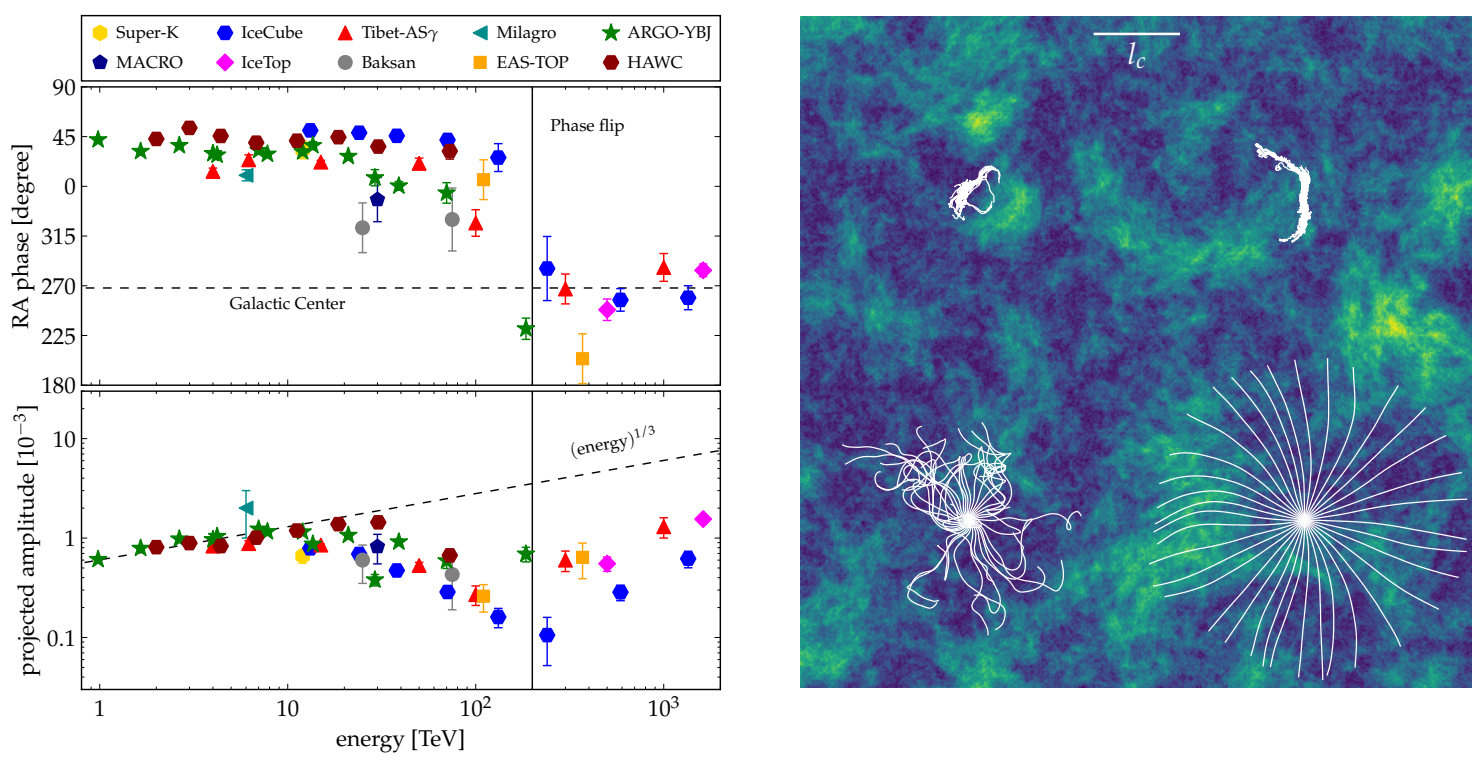

Figure 1: Left: Inferred phase and amplitude of the (equatorial) dipole anisotropy from recent measurements [9-12]. Open symbols indicate upper limits and the dashed horizontal line shows the RA phase of the Galactic Center. Right: Illustration of cosmic ray trajectories within $\Delta t=2 l_{c} / c$ prior to convergence at the indicated location. The different panels assume rigidities of $10,100,10^{3}$ and $10^{4} \mathrm{TV}$ from top-left to bottom-right and an average magnetic field stength of $4 \mu \mathrm{G}$. The $\mathrm{CR}$ arrival directions are assumed to lie within the $x y$-plane with 10 degrees spacing. The background shows the relative energy density of the turbulence at $z=0$.

are global properties of ensemble-averaged distributions. In standard diffusion theory, we can then study the angular-integrated PSD $n=n(t, \mathbf{r}) \equiv \int \mathrm{d} \hat{\mathbf{p}} f(t, \mathbf{r}, \mathbf{p})$ that satisfies a diffusion equation, $\partial_{t} n \simeq \nabla(\mathbf{K} \nabla n)$, where the diffusion tensor $\mathbf{K}$ can be expressed as

$$
\mathrm{K}_{i j}=b_{i} b_{j} \kappa_{\|}+\left(\delta_{i j}-b_{i} b_{j}\right) \kappa_{\perp},
$$

where $\mathbf{b}$ is a unit vector in the direction of the regular magnetic field. The leading-order PSD can then be written

$$
4 \pi f \simeq n-3 \hat{\mathbf{p}} \mathbf{K} \nabla n,
$$

i.e., the local PSD has a dipole anisotropy in the presence of a local gradient $\nabla n$.

It is important to realize that the dipole in Eq. (2) represents the anisotropy of the PSD averaged over spatial scales larger than typical diffusion scales. If we want to compare this to the observed dipole anisotropy of Fig. 1 we have to consider local effects of cosmic ray streaming. In the right panel of Fig. 1 we illustrate the path of cosmic rays in purely turbulent magnetic fields prior to their arrival at the observer. The different sets of trajectories all arrive within the $x y$-plane at equally spaced angular distances. We show the case of four representative rigidities. The trajectories of the top-left set of low-rigidity cosmic rays are tightly wound around the local magnetic field lines. Effectively, CR streaming becomes locally anisotropic even in the absence of a constant magnetic field [14]. This effect can not be accounted for by the structure of the diffusion tensor in Eq. (1). 
In [13] it was shown that the power spectrum of cosmic ray arrival direction induced by a non-vanishing background gradient, $\nabla n$, can be estimated by

$$
\frac{C_{\ell}}{4 \pi} \simeq \int \frac{\mathrm{d} \widehat{\mathbf{p}}_{1}}{4 \pi} \int \frac{\mathrm{d} \widehat{\mathbf{p}}_{2}}{4 \pi} P_{\ell}\left(\widehat{\mathbf{p}}_{1} \widehat{\mathbf{p}}_{2}\right) \lim _{T \rightarrow \infty} \Delta r_{1 i}(-T) \Delta r_{2 j}(-T) \frac{\partial_{i} n \partial_{j} n}{n^{2}},
$$

where $\Delta \mathbf{r}_{1}(t) \equiv \mathbf{r}_{1}(t)-\mathbf{r}_{1}(0)$ and $\dot{\mathbf{r}}_{1}(0)=\widehat{\mathbf{p}}_{1}$, etc. The power of the dipole anisotropy, $\ell=1$, can be expressed as

$$
\frac{C_{1}}{4 \pi} \simeq \mathrm{S}_{i j} \frac{\partial_{i} n \partial_{j} n}{n^{2}}
$$

where $\mathbf{S} \equiv \mathcal{K}^{T} \mathcal{K}$ and diffusion matrix,

$$
\mathcal{K}_{i j} \equiv \lim _{T \rightarrow \infty}\left\langle\widehat{p}_{i}(0) \Delta r_{j}(-T)\right\rangle_{\Omega},
$$

where the average $\langle\cdot\rangle_{\Omega}$ is over the different orientation of initial particle trajectories, $\widehat{\mathbf{p}}(\Omega)$. This formulation of the diffusion tensor coincides with the TKG one [15-17] if one trades the angular for an ensemble average over the magnetic configurations $\langle\cdot\rangle_{\mathrm{B}}$. In general, we have the identity $\langle\mathcal{K}\rangle_{\mathrm{B}}=\mathbf{K}$.

In the absence of a background magnetic field, we expect that the ensemble-averaged diffusion tensor approaches the isotropic form

$$
\mathrm{K}_{i j}=\delta_{i j} \kappa_{\text {iso }} .
$$

The isotropic diffusion scale $\kappa_{\text {iso }}$ can be estimated either via the magnetic ensemble average of Eq. (5) or, equivalently, via its integrated form,

$$
\kappa_{\text {iso }}=\lim _{T \rightarrow \infty}\left\langle\left\langle\Delta \mathbf{r}^{2}(-T)\right\rangle_{\Omega}\right\rangle_{\mathrm{B}} / 6 T .
$$

In these proceedings, however, we are interested in the effect of local anisotropies based on the random orientation of large scale fluctuations. One can see from Eq. (4) that the ensemble-averaged expectation value of the dipole power is determined by $\langle\mathbf{S}\rangle_{B} \neq \mathbf{K}^{T} \mathbf{K}$.

Since the matrix $\mathbf{S}$ is real and symmetric, we can parametrize it at any look-back time $T$ by its three real eigenvalues and three Euler angles that indicate the coordinate system where $\mathbf{S}$ becomes diagonal. By definition, the eigenvalues of $\mathbf{S}$ must be positive. Those modes of the local turbulence with a wavelength much larger than the gyroradius of the cosmic ray, will appear effectively as a local regular magnetic field direction, $\widehat{\mathbf{B}}$, that split the hierarchy of eigenvalues of $\mathbf{S}$ into one enhanced diffusion scale parallel to the magnetic field and two reduced diffusion scales. In general there are thus three different eigenvalues. Assuming a plane wave structure for the local magnetic field, we will therefore make the ansatz:

$$
\mathrm{S}_{i j} \simeq \kappa_{\mathrm{L}}^{2} b_{i} b_{j}+\kappa_{\mathrm{S} 1}^{2} v_{i} v_{j}+\kappa_{\mathrm{S} 2}^{2}\left(\delta_{i j}-b_{i} b_{j}-v_{i} v_{j}\right),
$$

where $\mathbf{b}$ is now the unit vector pointing into the direction of the effective local magnetic field and $\mathbf{v}$ a unit vector orthogonal to $\mathbf{b}$, which accounts for the local anisotropy of perpendicular diffusion in the volume-averaged realization of turbulence. Assuming that the direction of the cosmic ray gradient is not correlated with the local magnetic field direction, we arrive at

$$
\frac{\left\langle C_{1}\right\rangle_{\mathrm{B}}}{4 \pi} \simeq \frac{\left\langle\kappa_{\mathrm{L}}^{2}\right\rangle_{\mathrm{B}}+\left\langle\kappa_{\mathrm{S} 1}^{2}\right\rangle_{\mathrm{B}}+\left\langle\kappa_{\mathrm{S} 2}^{2}\right\rangle_{\mathrm{B}}}{3} \frac{(\nabla n)^{2}}{n^{2}}
$$


In the following, we will show by simulations that this has two effects. On one hand, the expectation value of the dipole power is enhanced compared to naive estimates using isotropic diffusion scale and shows a different scaling with rigidity. On the other hand, the anisotropy of the local diffusion tensor will lead to strong projection effects when determining the amplitude and phase of the dipole anisotropy via Eq. (4).

\section{Test-Particle Simulations}

In the following, we will study the ensemble averages of $\mathbf{K}$ and $\mathbf{S}=\mathcal{K}^{T} \mathcal{K}$ with the help of testparticle simulations in synthetic magnetic fields. We consider a static magnetic field configurations with a vanishing background field, $\mathbf{B}_{0}=0$, and $3 \mathrm{D}$ isotropic turbulence. The power spectrum of the isotropic turbulence, $\mathcal{P}(k)=k^{2} g(k)$, is normalized by the root-mean-square magnetic field as

$$
B_{\mathrm{rms}}^{2} \equiv\left\langle\delta \mathbf{B}^{2}\right\rangle=2 \int \mathrm{d} k \mathcal{P}(k)
$$

We assume a Kolmogorov spectrum without helicity $\left(\sigma(k)=0\right.$ and $\left.g(k) \propto k^{-11 / 3}\right)$ ranging between $k_{\min }$ and $k_{\max }$. An important characteristic of the turbulence is the coherence length, which we define as [18]. For $k_{\max } \gg k_{\min }$, the coherence length is then simply $l_{c}=L_{\max } / 5$.

In the following, we backtrack cosmic rays with rigidities from $1 \mathrm{TV}$ to $10 \mathrm{PV}$ through a 3D isotropic magnetic turbulence with root-mean-square field strength of $1 \mu \mathrm{G}$. The size of $k_{\min }=2 \pi / L_{\max }$ is set by the outer scale of the turbulence fixed at $L_{\max }=1.56 \mathrm{pc}$. In order to achieve a large dynamical range of the turbulence between $1.56 \mathrm{pc}$ and tens of AU, we set up the turbulent magnetic field as a nested grid [19]. We use six nested grids of $128^{3}$ grid points each so that it takes a relatively small memory space $\sim 0.3 \mathrm{~GB}$. In Fourier space, each grid covers a factor 8 in dynamical range, while the factor $128 / 8=16$ remaining is used for padding, and split the following: a factor 4 to develop large wavelength modes and avoid artificial anisotropy and a factor 4 to densify the modes in the dynamical range. With this set-up we cover a dynamical range of $2.6 \times 10^{5}$ with more than $1.5 \times 10^{5}$ modes per decade, spaced linearly, and ensure that the gyroradius $r_{g}$ of the propagated particle is at least ten times larger than the smallest wavelength of the turbulence.

The particle trajectories are evaluated with a customized version of CRPROPA [20] using the pre-encoded Boris-Push algorithm. For the integration we adopt the following prescription for the step size: $\delta_{\text {step }}=\min \left(l_{c} / 5,2 \pi r_{g} / 10\right)$ that enables us to compute $\mathrm{K}_{\text {iso }}$ with an accuracy below $2 \%$. We sample over 150 different turbulent magnetic field configurations. For each field configuration we uniformly sample $N_{\text {pix }}=12288 \mathrm{CR}$ orientations $\widehat{\mathbf{p}}_{i}(0)$ following the HEALPix parametrization $\left(n_{\text {side }}=32\right)$ [21], that we backtrack through the static magnetic field to find the initial position $\mathbf{r}(-T)$. Assuming that trajectories are following a uniform tiling of the unit sphere (such as the HEALPix parametrization), the diffusion matrix $\mathcal{K}$ can be estimated from

$$
\mathcal{K}_{i j}=\lim _{T \rightarrow \infty} \frac{1}{N_{\text {pix }}} \sum_{n=1}^{N_{\text {pix }}} \widehat{p}_{n i}(0) \Delta r_{n j}(-T),
$$

where, as before, $\widehat{p}_{n i}$ denotes the component $i$ of the vector $\widehat{\mathbf{p}}_{n}$, etc. The scales $\kappa_{L}$ and $\kappa_{S}$ can then be derived from the ensemble-averaged eigenvalues of $\mathbf{S}=\mathcal{K}^{T} \mathcal{K}$. 
Due to the discrete sampling of $N_{\text {pix }}$ trajectories, the expression for $\mathcal{K}$ is affected by simulation noise. We can estimate the noise level in each magnetic field configuration from the scrambled diffusion tensor

$$
\widetilde{\mathcal{K}}_{i j}(\xi)=\lim _{T \rightarrow \infty} \frac{1}{N_{\text {pix }}} \sum_{n=1}^{N_{\text {pix }}} \widehat{p}_{\xi_{n} i}(0) \Delta r_{n j}(-T),
$$

where the vector $\boldsymbol{\xi}$ corresponds to a random permutation of the tuple $\left(1, \ldots, N_{\text {pix }}\right)$. For individual magnetic ensembles, the symmetric matrix $\widetilde{\mathbf{S}}=\widetilde{\mathcal{K}}^{T} \widetilde{\mathcal{K}}$ has eigenvectors that do not align with those of the true $\mathbf{S}=\mathcal{K}^{T} \mathcal{K}$. However, the total sum of eigenvalues of $\mathbf{S}$ is invariant under random rotation. This allows us to correct for a noise bias from the total sum eigenvalues $\left\langle\kappa_{\mathrm{L}}^{2}\right\rangle_{\mathrm{B}}+\left\langle\kappa_{\mathrm{S} 1}^{2}\right\rangle_{\mathrm{B}}+\left\langle\kappa_{\mathrm{S} 2}^{2}\right\rangle_{\mathrm{B}}=$ $\operatorname{Tr}(\mathbf{S})-\operatorname{Tr}(\widetilde{\mathbf{S}})$.

\section{Results}

From the simulations we compute the time-dependent matrix $\mathbf{S}$ from which we extract the three eigenvalues $\kappa_{L}^{2}, \kappa_{S 1}^{2}$ and $\kappa_{S 2}^{2}$. In the following we introduce three diffusion lengths $\lambda_{i}$ (with $i \in[1,2,3])$ ordered by decreasing value and defined as $\lambda=3 \kappa / c$. In Fig. 2 we show the results from our simulations with test particles of rigidity $10 \mathrm{TV}$ propagating in a homogeneous turbulent magnetic field with root-mean-square $\delta B=1 \mu \mathrm{G}$ and outer-scale $L_{\max }=1.56 \mathrm{pc}$. The left panel shows the evolution of the ensemble average over 150 magnetic field realizations of the three eigenvalues $\hat{\lambda}_{i} \equiv\left\langle{\widehat{\lambda_{i}^{2}}}^{1 / 2}\right.$ with backtracking time is displayed with solid lines. We also show as dotted black lines the three eigenvalues $\lambda_{i}^{\text {iso }}$ corresponding to the conventional diffusion tensor $\kappa_{i j}(T)=\left\langle\Delta r_{i}(-T) \Delta r_{j}(-T)\right\rangle_{\Omega} / 2 T$. The value of $\lambda^{\text {iso }}$ corresponds to the average of these three eigenvalues.

For small backtracking times, typically smaller than one gyration in the mean magnetic field $\delta B$, the diffusion lengths increase linearly with time in accordance with a ballistic transport regime. After a transition period, which is slightly longer for $\lambda_{i}^{\text {iso }}$ than for $\hat{\lambda}_{i}$, the values reach a plateau corresponding to the convergence to the diffusive regime. We note that while the different $\lambda_{i}^{\text {iso }}$ all converge toward the same value, the values of $\hat{\lambda}_{i}$ differ significantly and span more than one order of magnitude: they encode the anisotropy of the local diffusion tensor. We estimate the asymptotic values and corresponding uncertainties by fitting $\hat{\lambda}_{i}$ above the time $T_{f}$ delineated by the vertical gray line where the largest eigenvalue $\hat{\lambda}_{1}$ flattens.

The fitting procedure and how we deal with the numerical noise will be detailed in [22]. The best-fit value of the asymptotic value and the uncertainties are shown with as the data points in the left panel of Fig. 2. The direct consequence of the eigenvalue hierarchy is a strong projection effect of the cosmic-ray gradient onto the direction of largest eigenvalue (see Eq. 4), the latter being aligned with the local magnetic field. Then a variation of the gradient orientation with the energy implies that the CR dipole varies with the energy, as proposed in [8].

We perform this analysis for each decade from rigidity $1 \mathrm{TV}$ to $10 \mathrm{PV}$. The evolution of the ratio of the eigenvalues with energy is shown in the right panel of Fig. 2. We note that both ratios $\hat{\lambda}_{2} / \hat{\lambda}_{1}$ and $\hat{\lambda}_{3} /$ widehat $\lambda_{1}$ increase with particle rigidity (for a fixed coherence length), and saturate to 1 above $r_{g} / l_{c} \simeq 1$. As illustrated by the right panel of Fig. 1 , this stems from the fact that for large Larmor radii, CRs are less sensitive to the local structure of the magnetic field and probe 

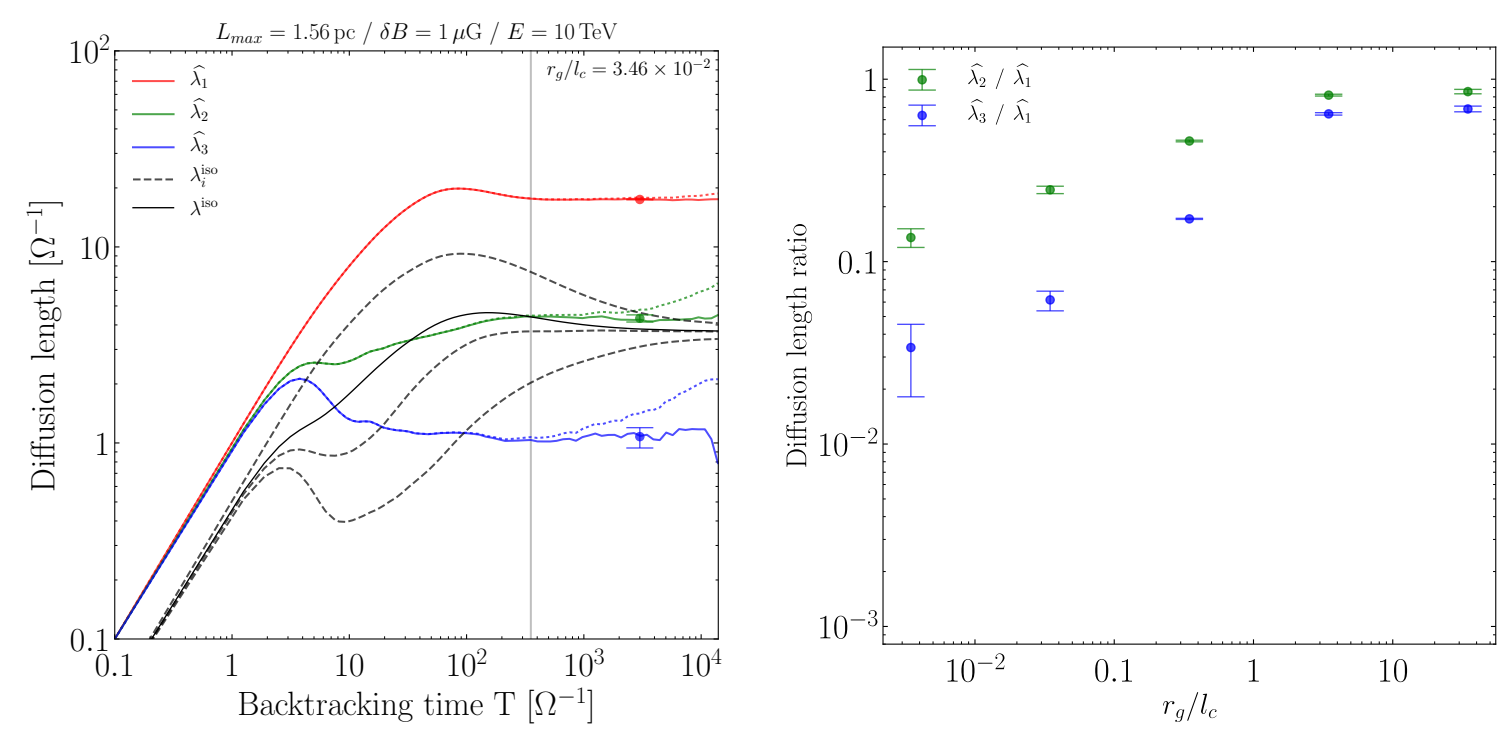

Figure 2: Left: The evolution of the ensemble averages of $\lambda_{i} \equiv\left\langle\lambda_{i}^{2}\right\rangle^{1 / 2}$ (with $\lambda_{i}^{2}$ eigenvalues of the matrix $\mathbf{S}=\mathbf{K}^{T} \mathbf{K}$ ) as a function of backtracking time is displayed with a dotted red, green and blue lines. We shown with a corresponding solid line or estimate $\widehat{\lambda}^{i}$ after noise correction. The vertical gray line indicates the time above which we perform the estimation of the steady eigenvalues shown with dots with corresponding uncertainties. For comparison we also show $\lambda_{\text {iso }}^{i}$ which correspond to the eigenvalues of the conventional diffusion tensor $\mathbf{K}_{0}$ are shown with black dotted lines. Finally, the mean of these eigenvalues $\lambda_{\text {iso }}$ (equals to $3 \kappa_{\text {iso }}$ defined Eq. 7) is shown with a solid black line. This result is based on ensemble-averaged quantities from a set of 150 magnetic field configurations with $n_{\text {sides }}=32$. Right : Evolution of the eigenvalues ratios with the particle Lamor radius.

uncorrelated perturbations. The local diffusion tensor $\mathcal{K}$ becomes isotropic. Note however that for small $r_{g} / l_{c}$, the diffusion length ratios are small and could lead to a projection effect of order $O(30)$. Among the other rigidity dependent effects (direction of the CR gradient and the effective local magnetic field), the evolution of the local diffusion lengths could provide an explanation for the increase of the dipole amplitude from $200 \mathrm{TeV}$.

In the later we focus on the ensemble average over 150 magnetic field realisations. However we are observing cosmic rays in a unique realisation, so it is important to assess the variations one could expect from one realisation to another. A detailed assessment will be provided in [22], where we show that the median of the distribution of the diffusion length behaves similarly to the mean. Defining the quantiles of this distribution indicates that, if the CR gradient becomes parallel to the direction of the smallest eigenvalue, the expected projection effect likely reaches $O(100)$ for the lower value $r_{g} / l_{c}$ studied.

\section{Summary and conclusion}

With the help of test-particle simulations we have devised a new methodology to study the local diffusion tensor $\mathcal{K}$ and its anisotropy. We have shown that in isotropic turbulence emerges a local anisotropy of the phase-space density originating from the local structure of the magnetic field. This local anisotropic diffusion naturally induces a projection effect of the local cosmic-ray gradient 
needed to explain the decrease with energy of the measured dipole amplitude and its alignment with the local magnetic field. Furthermore, the evolution of the local diffusion tensor with the particles energy show that it converges toward an isotropic tensor. This evolution might also drive the increase of the dipole amplitude above $200 \mathrm{TeV}$. This seminal study opens a new avenue towards the understanding the CR dipole anisotropy.

\section{Acknowledgements}

The authors acknowledge support from VILLUM FondEN under project no. 18994.

\section{References}

[1] J. R. Jokipii, ApJ 146 (Nov., 1966) 480.

[2] A. H. Compton and I. A. Getting, Physical Review 47 (June, 1935) 817-821.

[3] A. D. Erlykin and A. Wolfendale, Astropart.Phys. 25 (2006) 183-194, arXiv: astro-ph/0601290.

[4] P. Blasi and E. Amato, JCAP 1201 (2012) 011, arXiv: 1105.4529.

[5] M. Pohl and D. Eichler, Astrophys.J. 766 (2013) 4, arXiv: 1208.5338.

[6] P. Mertsch and S. Funk, Phys.Rev.Lett. 114 (2015) 021101, arXiv: 1408. 3630.

[7] N. A. Schwadron et al., Science 343 (Feb., 2014) 988-990.

[8] M. Ahlers, Phys. Rev. Lett. 117 no. 15, (2016) 151103, arXiv: 1605.06446.

[9] M. Ahlers and P. Mertsch, Prog. Part. Nucl. Phys. 94 (2017) 184-216, arXiv: 1612.01873.

[10] A. U. Abeysekara et al., Astrophys. J. 865 no. 1, (2018) 57, arXiv: 1805.01847.

[11] W. D. Apel et al., Astrophys. J. 870 no. 2, (2019) 91.

[12] A. Aab et al., (Pierre Auger Collaboration), Astrophys. J. 891 (2020) 142, arXiv: 2002.06172.

[13] M. Ahlers and P. Mertsch, Astrophys. J. Lett. 815 no. 1, (2015) L2, arXiv: 1506.05488.

[14] G. Giacinti, M. Kachelriess, and D. Semikoz, Phys. Rev. Lett. 108 (2012) 261101, arXiv: 1204.1271.

[15] G. I. Taylor, Proceedings of the london mathematical society 2 no. 1, (1922) 196-212.

[16] R. Kubo, Journal of the Physical Society of Japan 12 no. 6, (1957) 570-586.

[17] M. S. Green, The Journal of Chemical Physics 19 no. 8, (1951) 1036-1046.

[18] D. Harari, S. Mollerach, E. Roulet, and F. Sanchez, JHEP 03 (2002) 045, arXiv: astro-ph/0202362.

[19] G. Giacinti, M. Kachelriess, D. Semikoz, and G. Sigl, JCAP 1207 (2012) 031, arXiv: 1112.5599.

[20] R. Alves Batista, A. Dundovic, M. Erdmann, K.-H. Kampert, D. Kuempel, G. Müller, G. Sigl, A. van Vliet, D. Walz, and T. Winchen, JCAP 05 (2016) 038, arXiv : 1603.07142.

[21] K. Gorski et al., Astrophys.J. 622 (2005) 759-771, arXiv : astro-ph/0409513.

[22] Y. Genolini and M. Ahlers, in preparation. 